\title{
Live birth in a woman with recurrent implantation failure and adenomyosis following transfer of refrozen-warmed embryos
}

\author{
Somayyeh Safari, Azita Faramarzi, Azam Agha-Rahimi, Mohammad Ali Khalili \\ Research and Clinical Center for Infertility, Shahid Sadoughi University of Medical Sciences, Yazd, Iran
}

The aim was to report a healthy live birth using re-vitrified-warmed cleavage-stage embryos derived from supernumerary warmed embryos after frozen embryo transfer (ET) in a patient with recurrent implantation failure (RIF). The case was a 39-year-old female with a history of polycystic ovarian syndrome and adenomyosis, along with RIF. After ovarian hyperstimulation, 33 cumulus-oocyte complexes were retrieved and fertilized with conventional in vitro fertilization and intracytoplasmic sperm injection. Because of the risk of ovarian hyperstimulation syndrome, 16 grade $B$ and $C$ embryos were vitrified. After 3 and 6 months, 3 and 4 B-C warmed embryos were transferred to the uterus, respectively. However, implantation did not take place. Ten months later, four embryos were warmed, two grade B 8-cell embryos were transferred, and two embryos were re-vitrified. One year later, the two re-vitrified cleavage-stage embryos were warmed, which resulted in a successful live birth. This finding showed that following first warming, it is feasible to refreeze supernumerary warmed embryos for subsequent ET in patients with a history of RIF.

Keywords: Adenomyosis; Embryo; Live birth; Vitrification

\section{Introduction}

Many infertility patients still experience recurrent implantation failure (RIF), regardless of the immense strides forward in the field of assisted reproductive technology (ART) [1]. Implantation failure has been ascribed to various factors, such as uterine abnormalities [2], endometrial factors, and adenomyosis. Endometrial factors including submucous fibroids, endometrial polyps, endometrial hyperplasia, endometritis, and synechiae are also known as implantation failure causes. Adenomyosis is recognized by endometrial tissue within the myometrium that has the relevant quality with endometriosis. Several studies have confirmed that adenomyosis may interfere with

Received: May 7, 2016 · Revised: Jun 7, 2016 • Accepted: Jun 16, 2016

Corresponding author: Azam Agha-Rahimi

Research and Clinical Center for Infertility, Shahid Sadoughi University of Medical Sciences, Yazd 8916877391, Iran

Tel:+98-538224705 Fax:+98-3538247087 E-mail:Azam_Agharahimi63@yahoo.com

This is an Open Access article distributed under the terms of the Creative Commons Attribution Non-Commercial License (http://creativecommons.org/licenses/by-nc/4.0/) which permits unrestricted non-commercial use, distribution, and reproduction in any medium, provided the original work is properly cited. embryo implantation [3]. Moreover, an important step in ART is embryo cryopreservation, which contributes to improved pregnancy rates and reduces multiple pregnancies. Using once-frozen-thawed embryos, many healthy live births are achieved every year [4]. When all of the thawed embryos survive and supernumerary embryos are available for refreezing after frozen embryo transfer (ET), these embryos can be refrozen for future ET. Therefore, this approach can provide new opportunities, especially in cases with multiple implantation failure $[4,5]$.

The present report discusses a rare case with a history of RIF, fibroids, and adenomyosis in the uterus. She gave birth at 36 weeks to a healthy baby weighing $2.0 \mathrm{~kg}$ after ET of her refrozen-thawed cleavage-stage embryos.

\section{Case report}

\section{Patient history}

The case was a 39-year-old female with a 6-year history of primary infertility. She was referred to our infertility center with a diagnosis of 
polycystic ovarian disease. Vaginal sonography revealed diffusely enlarged uterus $(12.5 \times 13.5 \mathrm{~cm})$ with adenomyosis and three $1.5-\mathrm{cm}$ fibroids on the posterior, fundal and anterofundal uterine wall. The husband's sperm concentration was $25 \times 10^{6} / \mathrm{mL}$, with $40 \%$ progressive motility and $4 \%$ normal morphology (strict criteria).

\section{Ovarian stimulation, ovum pick-up and in vitro fertilization}

The patient was treated for ovarian stimulation with a long protocol. Pituitary function was suppressed by administration of $0.5 \mathrm{mg} /$ day intramuscular (IM) buserelin (Suprefact, Aventis, Frankfurt, Germany), starting in the luteal phase of the menstrual cycle. When the ultrasound showed inactive ovaries, buserelin was reduced to 0.25 $\mathrm{mg} /$ day IM and continued until the day of human chorionic gonadotropin (hCG) administration. Controlled ovarian hyperstimulation was initiated with $150 \mathrm{IU} /$ day recombinant follicle-stimulating hormone (Gonal F, Serono, Geneva, Switzerland) on day 2 of the menstrual cycle. Ovarian response was monitored by serial ultrasound examinations and serum estradiol levels, then the hCG dose was adjusted. A single dose of 10,000 IU hCG (Pregnyl, Organon, Oss, The Netherlands) was administered when at least two follicles reached a mean diameter of $18 \mathrm{~mm}$. Transvaginal oocyte retrieval was performed 34 to 36 hours after hCG administration with a 17-gauge needle (Cook, Queensland, Australia). A total of 33 cumulus-oocyte complexes (COCs), including 32 metaphase II (MII) oocytes and one germinal vesicle oocyte, were retrieved and incubated in culture medium at $37^{\circ} \mathrm{C}, 6 \% \mathrm{CO}_{2}$ for 3 hours. We performed insemination and intracytoplasmic sperm injection (ICSI) methods together according to the clinic's treatment plan. Twelve COCs were inseminated with 25,000 progressive spermatozoa per oocyte in $100 \mu \mathrm{L}$ droplets of G-IVF (Vitrolife, Gothenburg, Sweden), and 20 MIl oocytes underwent ICSI. All zygotes from in vitro fertilization (IVF) and the injected oocytes were washed twice and cultured in droplets of G1 (Vitrolife). On day 2 post-insemination, a total of 20 embryos at 4-6-cell stages were obtained (seven embryos from IVF and 13 embryos from IVF). Embryos were graded from $A$ to $B$, based on percent fragmentation and expected cell counts: grade $A$, equal-sized symmetrical blastomeres with no fragmentation; grade $B$, equal-sized symmetrical blastomeres with $<10 \%$ fragmentation; grade $C$, uneven blastomeres with fragmentation of no more than $50 \%$ of the blastomeres; grade $D$, fragmentation of more than $50 \%$ of the blastomeres.

\section{Vitrification of embryos}

Due to ovarian hyperstimulation syndrome (OHSS) risk, on day 2 after insemination 16 grade $B$ and C embryos (at 3-5 cell stages) were cryopreserved on four Cryotops (Kitazato BioPharma, Fujinomiya, Japan) by vitrification. Briefly, after a two-step loading with equilibration solution containing 7.5\% (v/v) ethylene glycol (EG) and 7.5\% (v/v) dimethyl sulfoxide (DMSO), and vitrification solution containing 15\% (v/v) EG, 15\% (v/v) DMSO, and $0.5 \mathrm{~mol} / \mathrm{L}$ sucrose, embryos were loaded onto the polyester sheet of Cryotop in a volume of $<0.1 \mu \mathrm{L}$. After loading, the media was removed to leave only a thin layer covering the embryos, and the sheets were quickly immersed into liquid nitrogen (LN2) and capped, then stored in a large container.

\section{Warming of embryos, embryo culture after warming, and ET}

After 3 and 6 months, three and four of the B-C grade embryos (at 6-8-cell stages) that had been warmed and cultured for 24 hours were transferred to the uterus, respectively. For warming, the protective cap was removed from the Cryotop while it was still submerged in LN2 and the sheet was immersed directly into
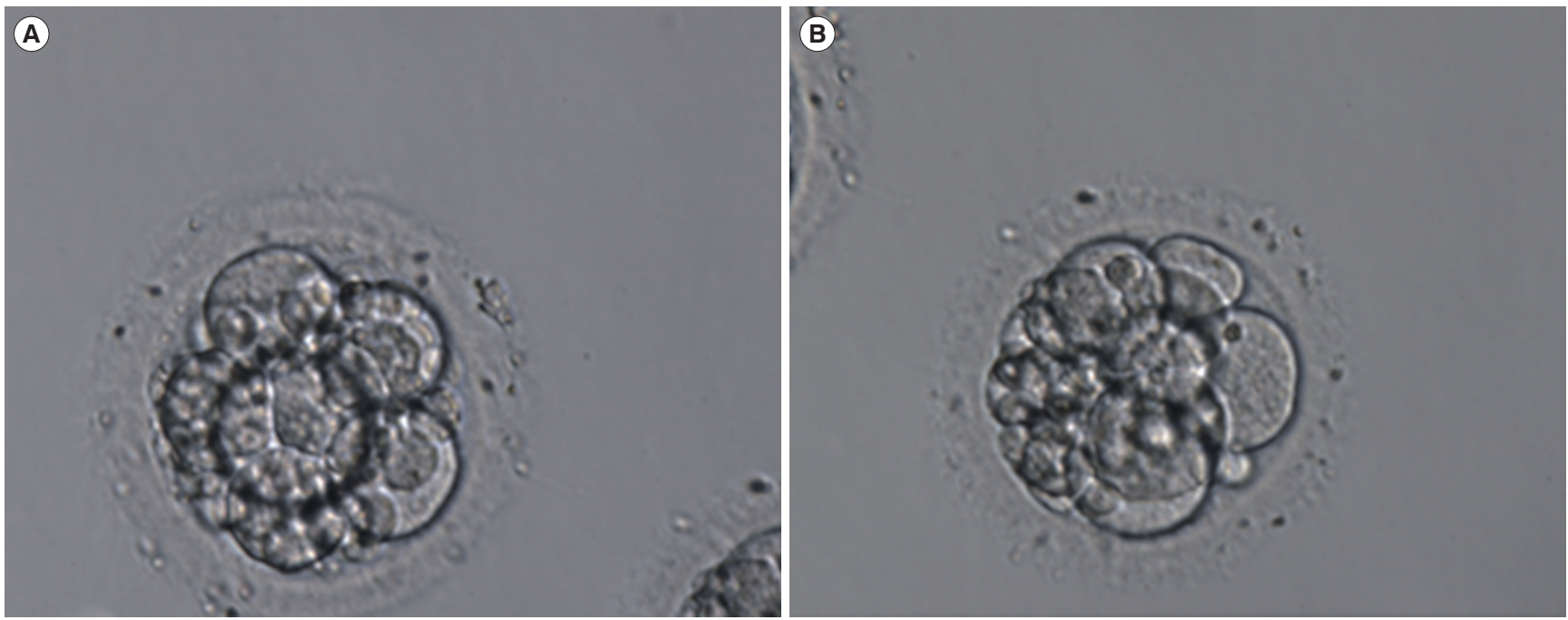

Figure 1. The embryos before transfer subjected to refreezing-warming at cleavage stage. (A) Grade C 7-cell embryo, (B) Grade C 8-cell embryo. 
warming solution containing $1 \mathrm{~mol} / \mathrm{L}$ sucrose. The embryos were sequentially incubated in diluents solution, washed three times in $\mathrm{G} 1$ v5 medium (Vitrolife) and incubated at $37^{\circ} \mathrm{C}$ and $6 \% \mathrm{CO}_{2}$ until ET. The patient was prepared for ET with oral estradiol (E2) to attain endometrial thickness $\geq 8 \mathrm{~mm}$ and a triple-line pattern on ultrasound scans. At that time, the patient was given $100 \mathrm{mg}$ of IM progesterone in oil daily and ET was preformed 3 days later under ultrasound guidance. However, the first and second frozen-warmed ET (FET) implantations failed.

\section{Re-vitrified, warmed ET, pregnancy, and live birth}

Ten months later, the third Cryotop was warmed. The embryos were subsequently cultured in $\mathrm{G} 1$ v5 medium at $37^{\circ} \mathrm{C}$ and $6 \% \mathrm{CO}_{2}$ overnight. Two grade $B$ and $C$ 8-cell embryos were transferred and two grade C 7- and 8-cell embryos were re-vitrified. The third attempt at FET failed again. One year after re-vitrification, the two revitrified cleavage embryos were warmed and both survived (Figure 1). On the day of warming, two 7- and 8-cell stage refrozen-warmed embryos were transferred to the uterus. FET preparation protocol applied to the patient as described previously. Then oral E2 and progesterone were continued until the 10th week of gestation. The pregnancy was confirmed, and she delivered a healthy female infant weighing $2 \mathrm{~kg}$ by caesarean section at 36 weeks of gestation.

\section{Discussion}

To the best of our knowledge, this is the first successful report on double-frozen cleavage stage ET in a 39-year-old patient with a history of RIF, fibroid, and adenomyosis in uterus. She did not have any experience of medication and/or treatment before or during FET. Twenty oocytes were fertilized after conventional IVF and ICSI. Sixteen of these embryos with good morphological quality were vitrified because of OHSS risk. Despite three unsuccessful attempts with vitrified-warmed embryos, the patient achieved pregnancy and live birth after the ET of double-frozen-warmed embryos. Previous studies have also presented success with the births of healthy babies after in vitro development of refrozen embryos [4-11]. In these cases, a slow freezing method or vitrification at other embryo development stages was used. However, we performed both vitrification protocols at the cleavage stage. To our knowledge, there have been no reports demonstrating that human embryos can be successfully refrozenwarmed at the cleavage stage in an advanced-age patient with RIF. Cryopreservation of supernumerary embryos during human IVF protocols provides a chance for patients to make repeated attempts at gestation after single-oocyte retrieval, ameliorating pregnancy rates and preventing the waste of valuable genetic material. Therefore, with technological progress, multiple cryopreservation of embryos has been contributed to treatment availability in ART [8]. There is an increased level of progesterone on the day of ovulation trigger, which was negatively associated with pregnancy rate in the initial fresh ET cycles. Therefore, freezing the embryos and performing ET subsequently can neutralize the problem [12]. There may be surplus warmed embryos available for re-cryopreservation when embryos are warmed and transferred in FET. These surplus embryos may be refrozen for later transfer to achieve successful pregnancies and live births. The refreezing process increases the cumulative pregnancy rate, while decreasing the risk of multiple gestation [13].

A new pathophysiological condition has been designated as RIF, described by multiple failures to achieve pregnancy after ET [14]. In certain cases, a unique condition due to unknown endometrial abnormalities, which do not even allow the initial steps of embryo implantation (apposition, attachment), can be described as RIF. A wide variety of reasons for RIF have been defined, such as maternal anatomic factors, including congenital uterine abnormalities, uterine fibroids, endometrial polyps, hydrosalpinges, adhesions, and endometriosis [2]. There is a considerable number of macrophages in the eutopic endometrium of patients with endometriosis, myomas, and adenomyosis [15]. Therefore, implantation potential may be impaired by adenomyosis [3]. The macrophage density increase induced by adenomyosis may cause a hostile immune environment for the implanting embryos. The appropriately activated macrophagesecreted cytokines tumor necrosis factor $a$ and interleukin 1, plus reactive oxygen (ROS) and nitrogen species are all potentially toxic to embryos. It has been shown that an elevated level of nitric oxide is related to unfavorable embryo development and poor pregnancy rates in the endometrial environment of patients with adenomyosis. In addition, endometrial biopsies taken from adenomyosis showed that this tissue is comprised of a high quantity of antioxidant enzymes, such as superoxide dismutase, catalase, and glutathione peroxidase, which are obvious signs of positional oxidative stress caused by excessive ROS production. The embryos directly damaged by these ROS and nitrogen species have been reported to augment local production of prostagrandin F2 $\alpha$ (PGF2 $\alpha$ ), causing an increase in myometrial contractility that can further disturb implantation [16].

We recommend freezing two embryos with average quality in each cryo-device. In the aforementioned case, four embryos were frozen on each Cryotop. On the third attempt, two embryos were transferred and two surplus embryos were refrozen. Ideally, one should not have surplus warmed early embryos, though this may occasionally occur. However refrozen-warmed embryos reached implantation and achieved live birth in a severe case with primary infertility. Therefore, this may alter the way we approach some FETs.

In conclusion, this case report asserts the position that repeated cryopreservation can be safely used for vitrification and suggests 
that human embryos could be re-vitrified. However, there is still a demand for prospective controlled studies on the obstetric and neonatal effects of refreezing and of vitrification in particular, despite these desirable results.

\section{Conflict of interest}

No potential conflict of interest relevant to this article was reported.

\section{References}

1. Das M, Holzer HE. Recurrent implantation failure: gamete and embryo factors. Fertil Steril 2012;97:1021-7.

2. Timeva T, Shterev A, Kyurkchiev S. Recurrent implantation failure: the role of the endometrium. J Reprod Infertil 2014;15:173-83.

3. Tremellen K, Russell P. Adenomyosis is a potential cause of recurrent implantation failure during IVF treatment. Aust N Z J Obstet Gynaecol 2011;51:280-3.

4. Mauri AL, Petersen CG, Oliveira JB, Baruffi RL, Al-Hasani S, Franco JG Jr. Embryos refrozen-thawed by vitrification lead to live births: case report. Middle East Fertil Soc J 2011;16:93-97.

5. Kumasako Y, Otsu E, Utsunomiya T, Araki Y. The efficacy of the transfer of twice frozen-thawed embryos with the vitrification method. Fertil Steril 2009;91:383-6.

6. Baker A, Check JH, Lurie D, Hourani C, Hoover LM. Pregnancy achieved with pronuclear-stage embryos that were cryopreserved and thawed twice: a case report. J Assist Reprod Genet 1996;13: 713-5.

7. Farhat M, Zentner B, Lossos F, Bdolah Y, Holtzer H, Hurwitz A. Successful pregnancy following replacement of embryos previously refrozen at blastocyst stage: case report. Hum Reprod 2001;16:337-9.
8. Smith LK, Roots EH, Dorsett MJ. Live birth of a normal healthy baby after a frozen embryo transfer with blastocysts that were frozen and thawed twice. Fertil Steril 2005;83:198-200.

9. Takahashi T, Araki Y. Successfully healthy baby delivery from human refrozen blastocyst embryos by vitrification. J Mamm Ova Res 2004;21:162-5.

10. Hashimoto S, Murata Y, Kikkawa M, Sonoda M, Oku H, Murata T, et al. Successful delivery after the transfer of twice-vitrified embryos derived from in vitro matured oocytes: a case report. Hum Reprod 2007;22:221-3.

11. Son WY, Lee SY, Chang MJ, Yoon SH, Chian RC, Lim JH. Pregnancy resulting from transfer of repeat vitrified blastocysts produced by in-vitro matured oocytes in patient with polycystic ovary syndrome. Reprod Biomed Online 2005;10:398-401.

12. Healy MW, Patounakis G, Connell MT, Devine K, DeCherney AH, Levy MJ, et al. Does a frozen embryo transfer ameliorate the effect of elevated progesterone seen in fresh transfer cycles? Fertil Steril 2016;105:93-9.e1.

13. Murakami M, Egashira A, Murakami K, Araki Y, Kuramoto T. Perinatal outcome of twice-frozen-thawed embryo transfers: a clinical follow-up study. Fertil Steril 2011;95:2648-50.

14. Simon A, Laufer N. Repeated implantation failure: clinical approach. Fertil Steril 2012;97:1039-43.

15. Khan KN, Kitajima M, Hiraki K, Fujishita A, Sekine I, Ishimaru T, et al. Changes in tissue inflammation, angiogenesis and apoptosis in endometriosis, adenomyosis and uterine myoma after GnRH agonist therapy. Hum Reprod 2010;25:642-53.

16. Tremellen KP, Russell P. The distribution of immune cells and macrophages in the endometrium of women with recurrent reproductive failure. Il: adenomyosis and macrophages. J Reprod Immunol 2012;93:58-63. 